\title{
Ab Initio Study of Screw Dislocations in Mo and Ta: A new picture of plasticity in bcc transition metals
}

\author{
Sohrab Ismail-Beigi ${ }^{\dagger}$ and T.A. Arias ${ }^{\ddagger}$ \\ $\uparrow$ Department of Physics, Massachusetts Institute of Technology, Cambridge, MA 02139 \\ $\ddagger$ Laboratory of Atomic and Solid State Physics, Cornell University, Ithaca, NY 14853
}

\begin{abstract}
We report the first $a b$ initio density-functional study of $\langle 111\rangle$ screw dislocations cores in the bcc transition metals Mo and Ta. Our results suggest a new picture of bcc plasticity with symmetric and compact dislocation cores, contrary to the presently accepted picture based on continuum and interatomic potentials. Core energy scales in this new picture are in much better agreement with the Peierls energy barriers to dislocation motion suggested by experiments.
\end{abstract}

The microscopic origins of plasticity are far more complex and less well understood in bcc metals than in their fcc and hcp counterparts. For example, slip planes in fcc and hcp metals are almost invariably close-packed, whereas in bcc materials many slip systems can be active. Moreover, bcc metals violate the Schmid law that the resistance to plastic flow is constant and independent of slip system and applied stress [1].

Detailed, microscopic observations have established that in bcc metals at low temperatures, long, low-mobility $\langle 111\rangle$ screw dislocations control the plasticity [2, 3]. Over the last four decades, the dominant microscopic picture of bcc plasticity involves a complex core structure for these dislocations. The key ingredient of this intricate picture is an extended, non-planar sessile core which must contract before it moves. The first such proposed structure respected the symmetry of the underlying lattice and extended over many lattice constants 4 . More recent and currently accepted theories, based on interatomic potentials, predict extension over several lattice constants and spontaneously broken lattice symmetry 2, 3, 5, 6]. While these models can explain the overall non-Schmid behavior, their predicted magnitude for the critical stress required to move dislocations (Peierls stress) is uniformly too large by a factor of about three when compared to experimental yield stresses extrapolated to zerotemperature 3 , [7].

We take the first $a b$ initio look at dislocation core structure in bcc transition metals. Although we study two metals with quite different mechanical behavior, molybdenum and tantalum, a consistent pattern emerges from our results which, should it withstand the test of time, will require rethinking the presently accepted picture. Specifically, we find screw dislocation cores with compact structures, without broken symmetry, and with energy scales which appear to be in much better accord with experimental Peierls barriers.

$A b$ initio methodology - Our ab initio calculations for Mo and Ta are carried out within the totalenergy plane-wave density functional pseudopotential approach [8, using the Perdew-Zunger [9] parameterization of the Ceperly-Alder 10 exchange-correlation energy. Non-local pseudopotentials of the KleinmanBylander form 11] are used with $s, p$, and $d$ channels. The Mo potential is optimized according to 12] and the Ta potential is from 13]. We use plane wave basis sets with energy cutoffs of 45 Ryd for Mo and 40 Ryd for Ta to expand the wave functions of the valence (outermost $s$ and $d$ ) electrons. Calculations in bulk show these cutoffs to give total system energies to within $0.01 \mathrm{eV} /$ atom. We carry out electronic minimizations using the analytically continued approach 14 within the DFT++ formalism 15.

To gauge the reliability of the pseudopotentials, Table 1 displays our $a b$ initio results for the materials' lattice constants and those elastic moduli most relevant for the study of $\langle 111\rangle$ screw dislocations. The tabulated moduli describe the long-range elastic fields of the dislocations $(K)$, the coupling of displacement gradients along the dislocation axis $z$ to core-size changes in the orthogonal $x, y$ plane $\left(c_{x x, z z}=\left(c_{11}+5 c_{12}-2 c_{44}\right) / 6\right)$, and the coupling of core-size changes to themselves in the plane $\left(c_{x x, x x}=\right.$ $\left(c_{11}+c_{12}+2 c_{44}\right) / 2$ and $\left.c_{x x, y y}=\left(c_{11}+2 c_{12}-2 c_{44}\right) / 3\right)$. 
These results indicate that our predicted core energy differences should be reliable to within better than $\sim 30 \%$, which suffices for the purposes of our study.

Preparation of dislocation cells - The cell we use for dislocation studies has lattice vectors $\vec{a}_{1}=$ $5 a[1,-1,0], \vec{a}_{2}=3 a[1,1,-2]$, and $\vec{a}_{3}=a[1,1,1] / 2$, where $a$ is the lattice constant. We call this ninetyatom cell the " $5 \times 3$ " cell in reference to the lengths of $\vec{a}_{1}$ and $\vec{a}_{2}$, and the Burgers vectors of all of the dislocations in our work are along $\vec{a}_{3}$. Eight $k$-points $k_{1}=k_{2}=\frac{1}{4}, k_{3} \in \pm\left\{\frac{1}{16}, \frac{3}{16}, \frac{5}{16}, \frac{7}{16}\right\}$ sample the Brillouin zone in conjunction with a non-zero electronic temperature of $k_{B} T=0.1 \mathrm{eV}$, which facilitates the sampling of the Fermi surface. These choices give total energies to within $0.01 \mathrm{eV} /$ atom.

Given the relatively small cell size, we wish to minimize the overall strain and the effects of periodic images. We therefore follow 16 and employ a quadrupolar arrangement of dislocations (a rectangular checkerboard pattern in the $\vec{a}_{1}, \vec{a}_{2}$ plane). This ensures that dislocation interactions enter only at the quadrupolar level and that the net force on each core is zero by symmetry, thereby minimizing perturbations of core structure due to the images. As was found in 16 and as we explore in detail below, we find very limited impact of finite-size effects on the cores when following this approach.

In bcc structures, screw dislocations are known to have two inequivalent core configurations, termed "easy" and "hard" [2, 5, 6]. These cores can be obtained from one another by reversing the Burgers vector of a dislocation line while holding the line at a fixed position. We produce cells with either only easy or only hard cores in this way. To create atomic structures for the cores, we proceed in three stages. First, we begin with atomic positions determined from isotropic elasticity theory for our periodic array of dislocations. Next, we relax this structure to the closest local energy minimum within the interatomic MGPT model for Mo[5]. Since we do not have an interatomic potential for Ta and expect similar structures in Ta and Mo[ $]$, we create suitable Ta cells by scaling the optimized MGPT Mo structures by the ratio of the materials' lattice constants. Finally, we perform standard $a b$ initio atomic relaxations on the resulting MGPT structures until all ionic forces in all axial directions are less than $0.06 \mathrm{eV} / \AA$.

Extraction of core energies - The energy of a long, straight dislocation line with Burgers vector $\vec{b}$ is $E=$ $E_{c}\left(r_{c}\right)+K b^{3} \ln \left(L / r_{c}\right)$ per $b$ along the line [17, where $L$ is a large-length cutoff, and $K$ is an elastic modulus (see Table 1) computable within anisotropic elasticity theory 18]. The core radius $r_{c}$ is a short-length cutoff inside of which the continuum description fails and the discrete lattice and electronic structure of the core become important. $E_{c}\left(r_{c}\right)$ measures the associated "core energy", which, due to severe distortions in the core, is most reliably calculated by ab initio methods.

The energy of our periodic cell contains both the energy of four dislocation cores and the energy stored outside the core radii in the long-range elastic fields. To separate these contributions, we start with the fact that two straight dislocations at a distance $d$ with equal and opposite Burgers vectors have an anisotropic elastic energy per $b$ given by $E=2 E_{c}\left(r_{c}\right)+2 K b^{3} \ln \left(d / r_{c}\right)$. Next, by regularizing the infinite sum of this logarithmically divergent pair interaction, we find that the energy per dislocation per $b$ in our cell is given by

$$
E=E_{c}\left(r_{c}\right)+K b^{3}\left[\ln \left(\frac{\left|\vec{a}_{1}\right| / 2}{r_{c}}\right)+A\left(\frac{\left|\vec{a}_{1}\right|}{\left|\vec{a}_{2}\right|}\right)\right] .
$$

The function $A(x)$ contains all the effects of the infinite Ewald-like sums of dislocation interactions and has the value $A=-0.598846386$ for our cell. Subtracting the long-range elastic contribution (the second term of (1D) from the total energy, we arrive at the core energy $E_{c}$.

To test the feasibility of this approach, we compare $E_{c}\left(r_{c}\right)$ for the MGPT potential as extracted with the above procedure from cells of two different sizes: the $5 \times 3$ cell and the corresponding $9 \times 5$ cell. (The MGPT is fit to reproduce experimental elastic moduli, so $K$ is given in Table1.) With the choice $r_{c}=2 b$, Table 2 shows that our results, even for the $5 \times 3$ cell, compare quite favorably with those of [5, 19], especially given that our $5 \times 3$ and $9 \times 5$ cells contain only ninety and 270 atoms respectively, whereas the cited works used cylindrical cells with a single dislocation and two thousand atoms or more. Given the suitability of the $5 \times 3$ cell, all $a b$ initio results reported below are carried out in this cell.

$A b$ initio core energies - Except for the Mo hard core, all the core structures relax quite readily from their MGPT configurations to their equilibrium $a b$ initio structures. The Mo hard-core configuration, however, spontaneously relaxes into easy cores, strongly indicating that the hard core, while meta-stable within MGPT by only $0.02 \mathrm{eV} / b \sqrt{19}$, is not stable in density functional theory. We do not believe that this instability is due to finite-size effects, which appear to be quite small for the reasons outlined previously.

Table 3 compares our ab initio results to available MGPT results for core energies in Mo and Ta. To 


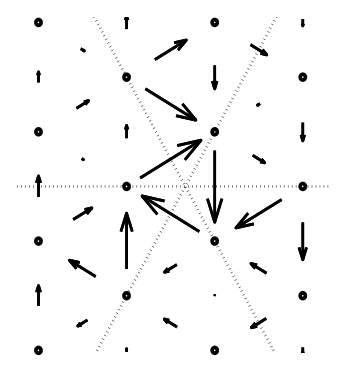

(a)

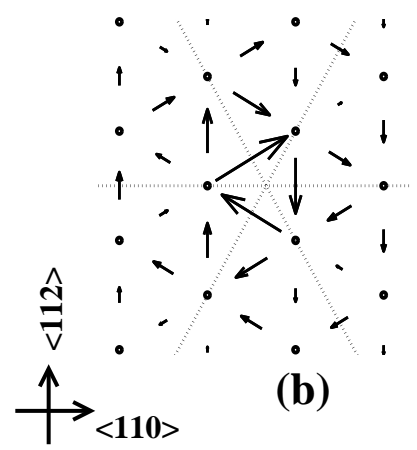

Figure 1: DD maps as found in the MGPT model for (a) easy, and (b) hard Mo dislocation cores. Dotted lines indicate axes of $C_{2}$ symmetry of the $D_{3}$ symmetry group.

make comparison with the MGPT, for the unstable Mo hard core we evaluate the $a b$ initio core energy at the optimal MGPT atomic configuration (column $\mathrm{AI}^{*}$ in Table 3). Note that, in computing hard-easy core energy differences, the long-range elastic contributions cancel so that these differences are much better converged than the absolute core energies.

Table 3 shows that the MGPT hard-easy core energy differences are much larger than the corresponding $a b$ initio values by approximately a factor of three. The accuracy of the elastic moduli of Table 1 combined with the high transferability of the localdensity pseudopotential approach, indicates that this factor of three is not an artifact of our approximations. We believe that the reason for this discrepancy is that the MGPT is less transferable. Having been forced to fit bulk elastic moduli and thus long-range distortions, the MGPT may not describe the short wavelength distortions in the cores with high accuracy. An examination of Mo phonons along [100] provides poignant evidence: the MGPT frequencies away from the zone center are too large when compared to experimental and band-theoretic values $[5]$ and translate into spring constants that are up to approximately three times too large.

The magnitude of the core energy difference has important implications for the magnitude of the Peierls energy barrier and Peierls stress for the motion of screw dislocations in Mo and Ta. In a recent Mo MGPT study 19, the most likely path for dislocation motion was identified to be the $\langle 112\rangle$ direction: the moving dislocation core changes from easy to hard and back to easy as it shifts along $\langle 112\rangle$. The energy barrier was found to be $0.26 \mathrm{eV} / b$, very close to the MGPT hard-easy energy difference itself. The fact that the $a b$ initio hard-easy energy differences in Mo and Ta are smaller by about a factor of three than the respective interatomic values suggests that the $a b$ initio energy landscape for the process has a correspondingly smaller scale. If so, the Peierls stress in Mo and Ta should also be correspondingly smaller and in much better agreement with the values suggested by experiments.

Dislocation core structures - Figure 1 shows differential displacement (DD) maps 2 of the core structures we find in our ninety-atom supercell when working with the interatomic MGPT potential for Mo. Our DD maps show the atomic structure projected onto the (111) plane. The vector between a pair of atomic columns is proportional to the change in the [111] separation of the columns due to the presence of the dislocations. The maps show that both easy and hard cores have approximate 3 -fold rotational $\left(C_{3}\right)$ point-group symmetry about the out-ofpage [111] axis through the center of each map. The small deviations from this symmetry reflect the weakness of finite-size effects in our quadrupolar cell. The hard core has three additional 2 -fold rotational $\left(C_{2}\right)$ symmetries about the three $\langle 110\rangle$ axes marked in the maps, increasing its point-group symmetry to the dihedral group $D_{3}$ which is shared by the underlying crystal. The easy core, however, shows a strong spontaneous breaking of this symmetry: its core spreads along only three out of the six possible $\langle 112\rangle$ directions. Our results reproduce those of [5, 19] who employed much larger cylindrical cells with open boundaries, underscoring the suitability of our cell for determining core structure. This symmetry-breaking core extension is that which has been theorized to explain the relative immobility of screw dislocations and violation of the Schmid law in bcc metals.

Figure 2 displays DD maps of our ab initio core structures. Contrary to the atomistic results, we find that the low-energy easy cores in Mo and Ta have full $D_{3}$ symmetry and do not spread along the $\langle 112\rangle$ directions. Combining this with the above results concerning core energetics, we have two examples for which our pseudopotentials are sufficiently accurate to disprove the conventional wisdom that generic bcc metallic systems require broken symmetry in the core to explain the observed immobility of screw dislocations.

Turning to the hard core structures, the $a b$ initio resuts for Ta show a significant distortion when compared to the atomistic core (contrast Figure $1 \mathrm{~b}$ and Figure 2b). As the $a b$ initio Mo hard core was unstable, we believe that this distortion of the Ta hard core suggests that this core is much less stable within density functional theory than in the atomistic po- 

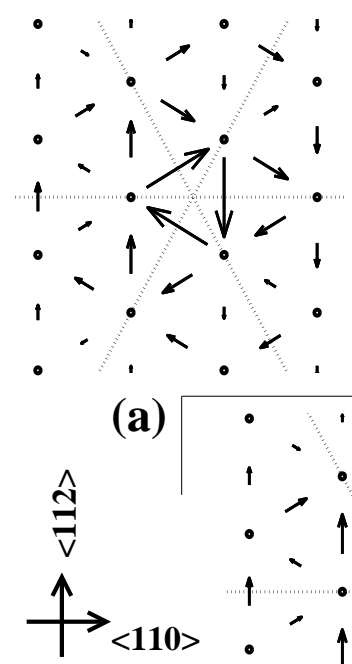

(a)
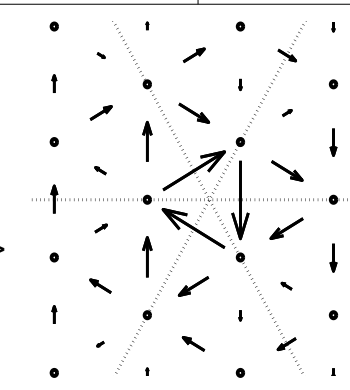

(c)

Figure 2: DD maps of the ab initio dislocation cores: (a) Ta easy, (b) Ta hard; and (c) Mo easy. Dotted lines indicate axes of $C_{2}$ symmetry of the $D_{3}$ symmetry group.

tentials.

To complete the specification of the threedimensional $a b$ initio structure of easy cores in Mo and Ta, Figure 3 presents maps of the atomic displacement in the (111) plane. The small atomic shifts, which are due entirely to anisotropic effects, are shown as in-plane vectors centered on the bulk atomic positions and magnified by a factor of fifty. To reduce noise in the figure, before plotting we perform $C_{3}$ symmetrization of the atomic positions about the [111] axis passing through the center of the figure. As all the dislocation cores in our study have a minimum of $C_{3}$ symmetry, this procedure does not hinder the identification of possible spontaneous breaking of the larger $D_{3}$ symmetry group. Our maps indicate that the easy cores in both Mo and Ta have full $D_{3}$ symmetry.

Recent high-resolution electron microscopy explorations of the symmetry of dislocations in Mo have focused on the small shifts in the (111) plane of columns of atoms along [111][20. This pioneering work reports in-plane displacements extending over a range much greater than the corresponding MGPT results and also much greater than what we find $a b$ initio. In 20 this is attributed to possible stresses from thickness variations and foil bending. We believe this makes study of the internal structure of the

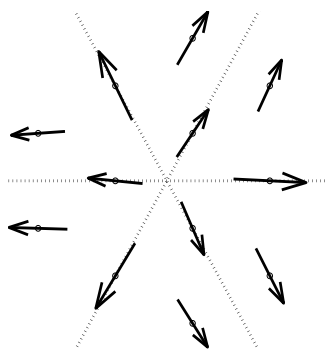

(a)

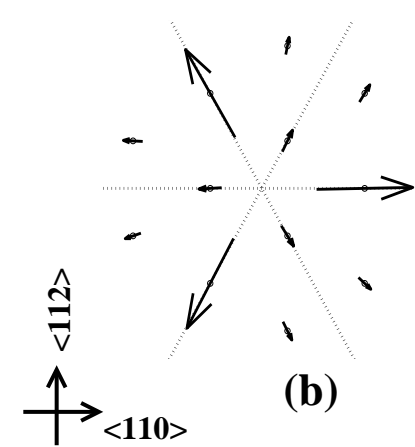

Figure 3: Planar displacement maps of the ab initio (a) Mo easy, and (b) Ta easy cores. Vectors show in-plane (111) atomic shifts and have been magnified by a factor of fifty. Dotted lines indicate the $C_{2}$ axes of the $D_{3}$ symmetry group.

core difficult, and that cleaner experimental results are required to resolve the nature of the symmetry of the core and its extension.

In conclusion, our first principles results show no preferential spreading or symmetry breaking of the dislocation cores and exhibit an energy landscape with the proper scales to explain the observed immobility of dislocations. Atomistic models which demonstrate core spreading and symmetry breaking, both of which tend to reduce the mobility of the dislocations, are well-known to over-predict the Peierls stress. The combination of these two sets of observations argues strongly in favor of much more compact and symmetric bcc screw dislocation cores than presently believed.

This work was supported by an ASCI ASAP Level 2 grant (contract \#B338297 and \#B347887). Calculations were run primarily at the Pittsburgh Supercomputing Center with support of the ASCI program and also on the MIT Xolas prototype SMP cluster. We thank members of the H-division at Lawrence Livermore National Laboratories for providing the Ta pseudopotential, the Mo MGPT code, and many useful discussions.

\section{References}

[1] E. Schmid, Proc. Int. Congr. Appl. Mech., 342 (1942).

[2] V. Vitek, Cryst. Lattice Defects 5 (1974), and references therein.

[3] M.S. Duesbery, Dislocations 1984, (CNRS, Paris 1984), p. 131. 
[4] Hirsch, P. B., Proc. 5th Int. Conf. Crystallography, Cambridge University, 139 (1960).

[5] W. Xu and J. A. Moriarty, Phys. Rev. B 54, 6941 (1996).

[6] J. A. Moriarty et al., J. Engr. Mater. and Tech. 121120 (1999).

[7] Z.S. Basinski and M.S. Duesbery, in Dislocation Modeling of Physical Systems, eds. M.F. Ashby et al., (Pergamon, Oxford 1980), p. 273.

[8] M.C. Payne, M.P. Teter, D.C. Allen, T.A. Arias, and J.D. Joannopoulos, Rev. Mod. Phys. 64, 1045 (1992).

[9] J. Perdew and A. Zunger, Phys. Rev. B. 23, 5048 (1981).

[10] D. M. Ceperly and B. J. Alder, Phys. Rev. Lett 45, 566 (1980).

[11] L. Kleinmann and D. M. Bylander, Phys. Rev. Lett. 48, 1425 (1982).

[12] A. Rappe, K. Rabe, E. Kaxiras, and J. D. Joannopoulos, Phys. Rev. B 41, 2127 (1990).

[13] C. Woodward, S. Kajihara, and L.H. Yang, Phys. Rev. B 57, 13459 (1998).

[14] T. A. Arias, M. C. Payne, and J. D. Joannopoulos, Phys. Rev. Lett. 69, 1077 (1992).

[15] S. Ismail-Beigi and T. A. Arias, Journal of Computational Chemistry, to be published.

[16] J. R. K. Bigger et al, Phys. Rev. Lett. 692224 (1992)

[17] J. P. Hirth and J. Lothe, Theory of Dislocations, John Wiley \& Sons, Inc.: New York, 1982.

[18] A. K. Head, Phys. Stat. Solidi 5, 51 (1964); Phys. Stat Solidi 6, 461 (1964).

[19] W. Xu and J. A. Moriarty, Comput. Mater. Sci. 9, 348 (1998).

[20] W. Sigle, Phil. Mag. A 79, 1009 (1999). 


\begin{tabular}{c||c|c|c||c|c|c}
\multicolumn{1}{l||}{} & \multicolumn{3}{c||}{ Mo } & \multicolumn{3}{c}{ Ta } \\
\hline & AI & Expt & Error & AI & Expt & Error \\
\hline \hline$a$ & 3.10 & 3.15 & $-1.6 \%$ & 3.25 & 3.30 & $-1.5 \%$ \\
\hline$K$ & 1.60 & 1.36 & $18 \%$ & 0.65 & 0.62 & $5 \%$ \\
\hline$c_{x x, z z}$ & 2.17 & 1.91 & $14 \%$ & 1.72 & 1.39 & $24 \%$ \\
\hline$c_{x x, x x}$ & 5.48 & 4.25 & $29 \%$ & 3.02 & 2.98 & $1.3 \%$ \\
\hline$c_{x x, y y}$ & 2.21 & 1.77 & $25 \%$ & 1.72 & 1.49 & $15 \%$
\end{tabular}

Table 1: Lattice constants $a(\AA)$ and elastic moduli (Mbar) for Mo and Ta based on ab initio pseudopotentials (AI) and experiments (Expt) 13.

\begin{tabular}{c||c|c|c}
$E_{c}(\mathrm{eV} / b)$ & $5 \times 3$ & $9 \times 5$ & Cylindrical [5, 19] \\
\hline \hline hard & 2.57 & 2.57 & 2.66 \\
\hline easy & 2.35 & 2.31 & 2.42 \\
\hline \hline$\Delta$ & 0.22 & 0.26 & 0.24
\end{tabular}

Table 2: Core energies for $r_{c}=2 b$ as predicted by the MGPT model. easy and hard refer to different core configurations. $\Delta$ is the hard-easy core energy difference.

\begin{tabular}{c||c|c|c|c|c}
\multicolumn{1}{c||}{} & \multicolumn{3}{c|}{ Mo } & \multicolumn{2}{c}{ Ta } \\
\hline$E_{c}(\mathrm{eV} / b)$ & MGPT & AI $^{*}$ & AI & MGPT [6] & AI \\
\hline hard & 2.57 & 2.94 & - & - & 0.91 \\
\hline easy & 2.35 & 2.86 & 2.64 & - & 0.86 \\
\hline \hline$\Delta$ & 0.22 & 0.08 & - & 0.14 & 0.05
\end{tabular}

Table 3: Core energies for $r_{c}=2 b$ for fully relaxed $a b$ initio cores (AI) and interatomic (MGPT) cores in the $5 \times 3$ cell. $\mathrm{AI}^{*}$ refers to ab initio core energies computed based on relaxed MGPT configurations as the ab initio Mo hard core is unstable. Ref.[6] only reported $\Delta$ for Ta. 